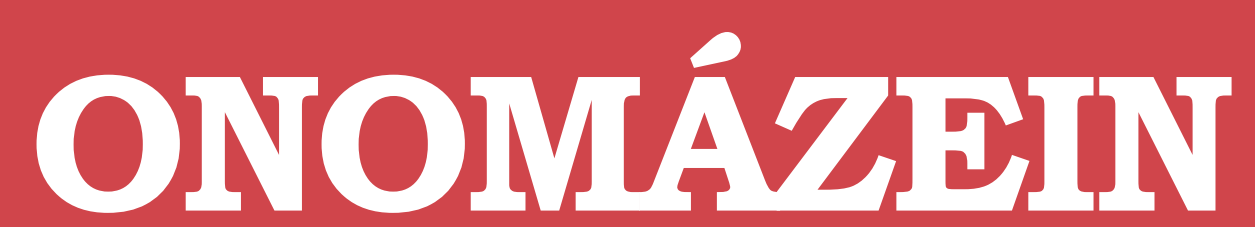

Revista de lingüística, filología y traducción
PONTIFICIA UNIVERSIDAD CATÓLICA DE CHILE FACULTAD DE LETRAS

\title{
Mantenimiento de una lengua minorizada: el caso del purépecha en Ichán y en Tacuro, Michoacán (México)
}

Maintenance of a minoritized language: the case of P’urhepecha in Ichán and Tacuro, Michoacán (Mexico)

\section{Felipe Canuto}

Universidad de Guanajuato

México

Número especial

Las lenguas amerindias en Iberoamérica: retos para el siglo XXI

2017
ONOMÁZEIN - Número especial

Las lenguas amerindias en Iberoamérica: retos para el siglo XXI (2017): 77-96 DOI: 10.7764/onomazein.amerindias.05

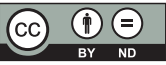

Felipe Canuto: Departamento de Estudios Culturales, Universidad de Guanajuato, campus León, México. | Correo electrónico: felipe.canuto@ugto.mx

Fecha de recepción: octubre de 2016 Fecha de aceptación: febrero de 2017 


\section{Resumen}

Históricamente, en México han confluido determinados factores que han propiciado el desplazamiento de las lenguas indígenas por el español. Sin embargo, en algunos pueblos la vitalidad de estas es todavía muy evidente, dado que las lenguas indígenas se han mantenido en el dominio lingüístico del hogary, por tanto, no se ha interrumpido la transmisión intergeneracional del idioma indígena.

La investigación ${ }^{1}$ que se presenta a continuación indaga acerca de los factores que podrían explicar que en las comunidades purépechas de Ichán y Tacuro, en Michoacán², la lengua indígena sea de empleo cotidiano entre la población en la mayoría de los ámbitos, sin olvidar el bilingüismo que caracteriza a la mayor parte de los hablantes y las presiones que se ejercen a favor del uso del español.

Palabras clave: México; mantenimiento lingüístico; lenguas indígenas; purépecha; Ichán; Tacuro.

\section{Abstract}

Historically, in Mexico several factors have caused the displacement of indigenous languages in favor of the use of Spanish. However, in some villages and towns, the vitality of indigenous languages is still very high owing to the fact that they are preserved at the family level and, therefore, the intergenerational transmission communication language at the family level is not interrupted.

1 Esta investigación se inscribe dentro del proyecto "Lenguas indígenas de México en contexto urbano", que fue financiado por el Programa para el Desarrollo Profesional Docente de la Secretaría de Educación Pública en la modalidad Apoyo a la Incorporación de Nuevos Profesores de Tiempo Completo.

2 Agradezco la colaboración de los vecinos, profesores, ministros de culto, personal de salud y de las jefaturas de Ichán y Tacuro, quienes compartieron conmigo sus saberes acerca de la cultura y lengua purépechas; la gratitud es también para quienes son originarios de estos pueblos y ahora radican en León, Guanajuato. 
The research presented here investigates the potential factors that explain the presence of the indigenous language in the daily life of the population in the purepecha communities Ichán and Tacuro (Michoacán) in most of the areas, despite bilingualism and the pressures exercised in favor of Spanish.

Keywords: Mexico; linguistic maintenance; indigenous languages; purepecha; Ichán; Tacuro. 


\section{Introducción}

Después de tres siglos de políticas lingüísticas coloniales que oscilaron entre la extinción y el mantenimiento de los idiomas indígenas, se llegó a un primer siglo de independencia en el cual debía resolverse, entre otros asuntos, el "problema del indio" y de la correspondiente ideología que consideraba perentoria la redención de un porcentaje de la población que, de acuerdo con la visión de la élite gobernante e intelectual, continuaba siendo india y, por tanto, un lastre para el deseado progreso de la nación mexicana. De esta manera, se llegó a la situación actual, en la que solo alrededor del 10\% de los habitantes del país habla un idioma indígena, quienes son también en su mayoría hablantes de español como segunda lengua.

Durante el periodo novohispano, a pesar del vaivén de las políticas lingüísticas implementadas, los idiomas amerindios gozaron de vitalidad, en gran medida en sus ámbitos de dominio original. En ciertos sectores sí se observó el cambio lingüístico en favor del casteIlano, como sucedió con los nobles indígenas que recibían educación en los conventos; sin embargo, la homogenización lingüística a favor del español no se generalizó debido, entre otros factores, a la práctica evangelizadora de los frailes, que enseñaron a los fieles la religión católica a través de sus propias lenguas (Wright, 2007).

El siglo XIX se inició con una revuelta dirigida por criollos que culminó con el proceso independentista, una independencia que en su devenir y en su proyecto de nación no incluyó a los indios y, desde luego, tampoco a sus lenguas. Pimentel (cit. en Villoro, 2014 [1950]: 185) señalaba que en el territorio mexicano existían dos pueblos diferentes que eran hasta cierto punto enemigos. En esta oposición, la escala jerárquica no favorecía a los indios y, aunque hubo ciertos intentos de posicionar sus idiomas en un plano de igualdad frente al español, lo anterior no se logró y el balance fue negativo; debido a que se privilegió el castellano en las interacciones públicas y este se oficializó en los ámbitos institucionales decimonónicos, se propició el desuso de las lenguas indígenas, con lo cual más de sesenta se extinguieron en esa centuria (Villavicencio, 2013: 119).

El siglo XX continuó con la política de asimilación de la población con cultura de raigambre mesoamericana e idioma distintos al español. La praxis de la ideología indigenista se vio reflejada en proyectos como la Casa del Estudiante Indígena, que pretendía "civilizar al indio" a costa de su identidad e integridad (Loyo, 1996); la Estación experimental, que estableció Moisés Sáenz $z^{3}$ en Carapan, Michoacán, para estudiar la realidad del indio "en el punto justo en que comienza a ser mexicano" con el fin de lograr su pronta incorporación a la "entidad nacional”

3 Entre los cargos públicos que desempeñó Sáenz se encuentran el de Subsecretario de Educación Pública y embajador. Organizó el Primer Congreso Indigenista Interamericano en 1940. 
(1992 [1936]); y los Centros Coordinadores con su programa de "aculturación inducida”, en la cual participaban agentes de las culturas dominante y de las subordinadas (INI, 1962).

Los proyectos citados contribuyeron a la pérdida de cultura e identidad indígenas; uno de los elementos más significativos (y distintivos), la lengua, fue de los que más embates sufrió por parte de los precursores de la unidad nacional en su búsqueda de una homogeneidad étnica y cultural. Aunque en el discurso se consideraba la particularidad y valía de lo indígena, en la práctica se realizaba lo contrario. Desde el siglo XIX la intención era una y la práctica otra. Pimentel4, por ejemplo, consideraba que debía procurarse que los indios "olvidasen sus costumbres y hasta su idioma mismo, si fuere posible. Sólo de ese modo perderán sus preocupaciones y formarán con los blancos una masa homogénea, una nación verdadera" (cit. en Villoro, 2014 [1950]: 194).

Con la llegada del siglo XXI se reconoció en la Constitución Política de los Estados Unidos Mexicanos (2001) la composición pluricultural del país, sustentada originalmente en los pueblos indígenas, y se promulgó la Ley General de Derechos Lingüísticos de los Pueblos Indígenas (2003); no obstante, debido al desconocimiento general que tenía la población sobre las lenguas indígenas y a la falta de apoyo por parte de las autoridades gubernamentales se repitieron las prácticas de discriminación por motivos de origen étnico y lingüístico (Canuto, 2013). En la actualidad, de acuerdo con el Instituto Nacional de Estadística, Geografía e Informática (INEGI)5, se hablan 72 idiomas; según el Instituto Nacional de Lenguas Indígenas $\left(\right.$ INALI) ${ }^{6}$ se trata de 364 variedades lingüísticas. De manera general, se puede señalar que uno de los factores que ha sido determinante en la extinción de los idiomas indígenas ha sido la política de castellanización, forzosa y excluyente, que se ha llevado a cabo con diferentes métodos y agentes desde la época colonial (Brice, 1992 [1970]). La obligatoriedad del aprendizaje del idioma español y la restricción de hablar el propio fueron determinantes para la pérdida de las lenguas indígenas.

Por consiguiente, se ha reducido, gradualmente, el número de indígenas que, como señala Aguirre Beltrán, han pasado "a integrarse, individualmente, en el grupo mestizo dominante" (1992 [1976]: 61). En el transcurso de cinco siglos las lenguas indígenas de México pasaron de

4 Francisco Pimentel fue un reconocido lingüista del siglo XIX, quien describió, analizó, comparó y clasificó las lenguas indígenas de México; entre sus textos se encuentra el Cuadro descriptivo y comparativo de las lenguas indigenas de México o tratado de filología mexicana.

5 "Hablantes de lengua indígena en México", en http://cuentame.inegi.org.mx/poblacion/lindigena. aspx?tema=p\#uno, fecha de consulta: 05 de mayo de 2016.

6 "Catálogo de las lenguas indígenas nacionales: variantes lingüísticas de México con sus autodenominaciones y referencias geoestadísticas", en http://www.inali.gob.mx/pdf/CLIN_completo.pdf, fecha de consulta: 05 de mayo de 2016. 
ser mayoritarias a minoritarias y, además, minorizadas, pues en la asimetría sociocultural que se estableció desde el siglo XVI se consideraron en una posición superior quienes se comunicaban en español y eran portadores de la cultura occidental7. Así, por ejemplo, durante la época novohispana las lenguas amerindias eran mayoritarias en cuanto al número de sus hablantes, pero minorizadas en calidad, en tanto que el español era minoritario por el reducido número de hablantes, pero mayorizado por el prestigio que este grupo detentaba. Y este mecanismo es el que ha permitido que el español de lengua minoritaria se haya convertido en mayoritaria y mayorizada, y, a la inversa, el náhuatl, lengua del imperio mexica, de mayoritaria y mayorizada se ha vuelto minoritaria y minorizada.

En una situación de asimetría sociocultural, las relaciones interétnicas no se circunscriben únicamente al número de los miembros de un determinado grupo sociocultural, sino, sobre todo, a la jerarquía que se instaura entre ellos y a partir de la cual se precian sus expresiones. De ahí que uno de los resultados que se producen es la pérdida del idioma de quienes ocupan el nivel bajo en la escala de valores sociales compartidos, porque se llega a naturalizar la idea de la superioridad de unos y la inferioridad de otros. Son numerosos los casos de desplazamiento lingüístico que se han producido en México. Por ejemplo, Zimmermann (2010) muestra cómo en Cardonal, Hidalgo, durante el siglo XX el otomí ha sido gradualmente reemplazado por el español: en una primera fase, de una situación de monolingüismo, se pasó a una de diglosia virtual con la incorporación del pueblo al contexto nacional; en la segunda, se produjo diglosia parcial con bilingüismo parcial de duración corta en ciertos dominios; en la tercera, se observó un incremento en el uso del castellano y diglosia sustitutiva; finalmente, en la cuarta, el español se adquirió como primera lengua por la población local y terminó por instaurarse como la de mayor empleo en el ámbito del hogar, familiar.

En el caso de los indígenas que han migrado a la ciudad el panorama, en general, es semejante al que acontece en los pueblos de origen: el desplazamiento de la lengua indígena se observa también con el paso de las generaciones y en tres de estas se puede producir la sustitución lingüística, como es el caso de los otomíes de Santiago Mexquititlán, Querétaro, que llegaron a la ciudad de México en la segunda mitad del siglo XX: Ios primeros que migraron eran monolingües (sobre todo las mujeres) o bilingües incipientes; sus hijos, que llegaron siendo niños o nacieron en la ciudad, se hicieron bilingües por el contacto con el idioma de

7 En este trabajo se diferenciará también entre lengua minoritaria y minorizada, por un lado, y mayoritaria y mayorizada, por otro. Una lengua minoritaria o una mayoritaria lo será por el porcentaje de hablantes que posea en relación con el total de la población en un determinado territorio o nación; por su parte, la minorización o mayorización de un idioma estará en función del estatus que se le otorgue en la jerarquía lingüística por los grupos de idioma en contacto. 
sus padres (cuando estos lo hablaban cotidianamente) y con el castellano de los citadinos y de la escuela; sus nietos, que nacieron cuando ya no se empleaba el otomí en el ámbito del hogar, aprendieron español como primera y única lengua, y del idioma indígena solo conocen ciertas fórmulas y léxico básicos (Canuto, 2015).

Debido a la discriminación de que son objeto los indígenas en México y al consecuente desprestigio de sus lenguas, el español se convierte paulatinamente en idioma mayorizado y, al mismo tiempo, se engruesan las cifras que lo convierten en mayoritario. Entre la literatura sociolingüística que aborda el desplazamiento y los factores que lo producen se encuentran los textos de Zimmermann (1992, 2010), Hekking (1995) o Terborg y García-Landa (2011).

Sin embargo, y a pesar de las situaciones mencionadas acerca de la minorización de las lenguas y los muchos casos de desplazamiento (y muerte) de estas, la historia lingüística del país también presenta casos, como el de algunos pueblos purépechas, donde el idioma indígena es de uso cotidiano en todos los ámbitos de comunicación y en los cuales no parecen cumplirse las reglas en cuanto a las condiciones que propician la pérdida de un idioma. Así parece suceder con el caso del purépecha en Santa Fe de la Laguna, Michoacán (Rico, 2010) o con Ichán y Tacuro, también Michoacán, que contrastan con lo que sucede, de manera general, no solo en México, sino a nivel mundial con los idiomas minoritarios y minorizados (Uranga y Magaña, 2008). En estos dos pueblos la vitalidad de la lengua purépecha es notoria, aunque en la cabecera municipal, Chilchota, el idioma indígena haya sido desplazado por el español.

Con estas premisas, el objetivo de este trabajo es presentar un estudio de caso en los dos lugares citados para analizar algunos de los factores que pueden barajarse para explicar el mantenimiento de la lengua amerindia, a pesar de las situaciones históricas, sociales y económicas que han sido adversas para sus hablantes.

\section{El contexto de una lengua minorizada en México}

Las relaciones interétnicas en México se caracterizan por una oposición entre indígenas y mestizos que remite, incluso, al plano geográfico (Navarrete, 2004: 7); tal y como se ha visto anteriormente, en términos generales, la situación de los indígenas en México hasta el siglo XXI ha sido de discriminación, lo cual se encuentra en estrecha relación con la pérdida de su cultura. De acuerdo con la "Primera encuesta nacional sobre discriminación en México", realizada por el Consejo Nacional para Prevenir la Discriminación (CONAPRED) en 2005, los indígenas aparecían en el cuarto lugar entre los más discriminados en el país, lo cual se reflejaba en menores oportunidades laborales y escolares y en peor nivel de vida; además, no se res- 
petaban sus derechos fundamentales ${ }^{8}$. En la "Segunda encuesta..." del CONAPRED de 2010 se sigue mencionando que la discriminación es el principal problema de los grupos indígenas?.

Según el texto Percepción de la imagen del indígena en México, cuando a los entrevistados (no indígenas o no identificados como tales) se les mencionaba la palabra "indígena”, resonaban ideas de pobreza y discriminación, además de marginación, atraso, diferencia, fealdad o ignorancia, entre otras (CDI, 2006: 13, 18). La probable vecindad con indígenas provocó reacciones de rechazo, lo cual también se observa en la "Primera encuesta..." del CONAPRED, pues en esta el $40 \%$ de los entrevistados dijo estar dispuesto a organizarse y no permitir que se establecieran cerca de su comunidad.

En lo que concierne particularmente a las lenguas, en una encuesta denominada "Percepción sobre las comunidades indígenas de México"10, el 68\% de los entrevistados respondieron que estaban en desacuerdo con que los indígenas hablasen solo su idioma. Aunque en esta muestra no se presenta información cualitativa, se observa que en la base de esta apreciación se encuentran los aspectos negativos con que se asocia a lo indígena: escasa o nula educación (escolar oficial), atraso, pobreza y poca confianza"1. De hecho, atendiendo a la "Primera encuesta..." del CONAPRED, en el imaginario de la población mexicana continúa arraigada la idea de que los indígenas, por sus "características raciales”, están limitados y la única manera que tienen para salir de la pobreza es "no comportarse como indígenas".

Una pregunta que surge entonces es ¿a partir de cuándo los indígenas dejan de ser sujetos de discriminación? Solo se esboza una posible respuesta. De acuerdo con las historias de vida de los indígenas que se han recopilado y de un análisis de estas a priori, el estigma desaparece cuando manejan los códigos de los mestizos; es decir, cuando hablan español en un nivel considerado estándar (su habla no presenta interferencias con la lengua indígena) y, en general, cuando comparten los rasgos culturales más notorios, que suelen ser diacríticos. Por ejemplo, quienes nacen en la ciudad o llegan a ella siendo niños socializan y viven de acuerdo con el mundo citadino y, por tanto, puede pasar desapercibida su condición biológica de indígenas; en cambio, sus padres y abuelos, debido a la diferencia cultural, notoria o en cierto

8 "Primera encuesta nacional sobre discriminación en México", en http://www.conapred.org.mx/ userfiles/files/Encuesta_Nal_sobre_discriminacion_resumen.pdf, fecha de consulta: 01 de mayo de 2016.

9 "Segunda encuesta nacional sobre discriminación en México", en http://www.conapred.org.mx/ userfiles/files/Enadis-2010-RG-Accss-002.pdf, fecha de consulta: 01 de mayo de 2016.

10 "Percepción sobre las comunidades indígenas de México", en http://www.parametria.com.mx/carta_parametrica.php?cp=4792, fecha de consulta: 05 de mayo de 2016.

11 "Cómo percibimos a los indígenas", en http://www.parametria.com.mx/carta_parametrica. php?cp=4688, fecha de consulta: 05 de mayo de 2016. 
grado evidente, padecieron escarnio y violencia que fueron disminuyendo conforme avanzó su asimilación o apropiación de la cultura mestiza.

Lo señalado anteriormente sirve como marco de referencia en el caso mexicano para observar que la minorización de las lenguas se encuentra en relación con el estatus de sus hablantes en la "sociedad nacional”. La mayorización o minorización de un idioma está determinada por el contexto social del grupo que lo porta: la valoración o el desprestigio de este suele corresponderse con el estatus jerárquico en el que se posiciona su lengua. En una relación asimétrica, como la que se observa en México, las culturas y las lenguas indígenas son consideradas inferiores y, en consecuencia, en la oposición que se establece se les califica con poco o ningún valor, o sólo sirven para fines folcloristas o de atracción turística.

Las lenguas son entes abstractos que no luchan entre ellas, sino que son sus hablantes quienes establecen una relación antagónica, pero en ningún caso por motivos meramente lingüísticos. Lo que se entiende por "conflicto lingüístico" es, más bien, una pugna entre grupos sociales que se traslada al ámbito de los idiomas (Ninyoles, 1975: 67): el idioma puede emplearse como pretexto para aparentar otra razón diferente a la que sustenta la intención de dominio; una de las formas mediante las cuales se busca justificar y legitimarlo es mediante la cientifización o la argumentación racional acerca de los supuestos beneficios que conlleva aprender el idioma del grupo dominante o, en el caso contrario, la dificultad que entraña la lengua de los dominados para llevarlos a la civilización ("enseñarles los misterios de la religión cristiana”, se decía en la época colonial), entre otras posibles verdades aceptadas y naturalizadas.

Cuando se resuelve un conflicto lingüístico, por cualquier medio, se coloca en un rango superior a un grupo y en uno inferior a otro; de igual manera, en el caso que aquí se trata, se ubica a los idiomas en un nivel determinado de acuerdo con la jerarquía lingüística que se instaura por los vencedores. La ideología de la superioridad llega a ser aceptada por los dominados debido a las situaciones que se propician para afianzarla y demostrarla, y aceptan su condición de subordinados: se minorizan a sí mismos y a sus lenguas y, de esta manera, se despeja el camino que los conducirá hacia la minoría numérica, pues gradualmente serán cada vez más los que no hablen su idioma y no lo transmitan a sus descendientes, y menos los que deseen perpetuarlo a pesar del sentimiento positivo hacia él como herencia de sus antepasados.

Entre las estrategias que se emplean en un conflicto lingüístico para minorizar una lengua y mayorizar otra se encuentran el desprestigio y la estigmatización de sus hablantes para colocarlos en situación de desventaja e inferioridad respecto del grupo que pretende ejercer el domino (Zimmermann, 1992). Así, las lenguas, en su calidad de mayorizadas o minorizadas, aluden al estatus sociocultural (incluso económico) de la comunidad que las emplea, a la posición que ocupan en la escala de valores instaurada a partir de la relación de poder, la cual es independiente de la mayoría o minoría en términos cuantitativos. 
A partir del establecimiento de una relación entre dos o más grupos en la cual uno logra acceder a una posición dominante, los elementos culturales de los subordinados se precian con minusvalía. Así, a las lenguas de los dominados se las considera, en general, inferiores, no se les concede la "calidad de idioma" (o se dice que existen idiomas superiores e inferiores), son "dialectos" en sentido peyorativo y a quienes los hablan se les señala como ignorantes, atrasados e incivilizados, como sucede en el caso de los indígenas en México; el compendio de lo anterior se traduce en expresiones populares del tipo "son dialectos de tribus". Calvet (2005 [1974]: 68) ha señalado también esta oposición creada entre el "civilizado" y el "salvaje" y ha aludido a las respectivas denominaciones que reciben sus lenguas.

La minorización efectiva de un idioma se produce cuando sus propios hablantes aceptan y se convencen de su poca utilidad lingüística ("las lenguas indígenas no son aptas para la ciencia", se dice, por ejemplo), del estigma social que representa (es una marca negativa que se debe superar o, cuando menos, ocultar) y del impedimento socioeconómico que consideran inherente a él y que les impone limitaciones (se debe hablar español para ascender en la escala social o, como han mencionado algunos, “¿para qué me sirve hablar lengua indígena?"), entre otros aspectos. Y, por estos motivos, las lenguas indígenas dejan de escucharse en los ámbitos cotidianos y de transmitirse a sus descendientes; en casos más extremos, existe oposición de los mismos miembros del grupo étnico para que otros las empleen y enseñen.

Ante esta situación se llega a la posibilidad de ser indígena, dejar de serlo o vivir entre los dos mundos y emplear los respectivos idiomas o no. En muchos pueblos la gente que migra a las ciudades ha optado por amestizarse. La posibilidad de acceder a los bienes y servicios del estado (entre ellos la educación), el deseo del ascenso socioeconómico y la necesidad de comunicarse con una mayoría hispanohablante al salir de la comunidad, entre otros factores, han sido determinantes para la adquisición del español. Indudablemente, en relación con la minorización de una lengua y la mayorización de otra se encuentra, por un lado, la condición nuclear o instrumental que se otorgue a las mismas por sus hablantes, es decir, la importancia que se le adjudique desde el ámbito doméstico hasta el plano social, ya sea como medio de expresión cotidiana en todos los contextos o solo en las interacciones con los ajenos; por otro lado, se ejerce presión y se propician condiciones desde las instituciones y la sociedad para que se olvide y no se enseñe el idioma propio y se adquiera el de prestigio, y la comunidad lingüística minorizada se vea forzada a adoptar y adaptar este último.

Una primera posibilidad en cuanto a su ubicación en los niveles de la cultura es, como ha sucedido en muchos casos, el posicionamiento del español en el núcleo y la sustitución de la lengua indígena por este debido a la minorización efectiva de que ha sido objeto por sus propios hablantes; una segunda es el bilingüismo por motivos meramente instrumentales, funcionales, y, aunque ambos idiomas conllevan un estatus nuclear, están diferenciados en las funciones: el idioma indígena poseerá dominio en sus propios contextos (entendidos 
como los espacios sociales y geográficos donde se encuentren los hablantes del mismo) y el español estará reservado para lo ajeno, para la interacción con los de fuera.

Sin embargo, la función principal de la lengua estará en relación, sobre todo, con su aspecto valorativo y apreciativo (en términos de ser considerada raíz, herencia y futuro) porque es parte fundamental de la identidad de una comunidad que, de esta manera, se diferencia entre el grueso de la población amestizada. Por tanto, a pesar de que una lengua tenga una posición minorizada en el plano nacional, si en sus dominios goza de mayorización estará asegurado su mantenimiento durante el tiempo en que sus hablantes consideren que la valía de su idioma no depende de factores extraculturales. Si no se aúnan minorización externa e interna, no surtirá una minorización efectiva.

\section{Metodología de la investigación}

Los resultados que se presentan en este texto son parte de una investigación que, en su conjunto, se lleva a cabo con indígenas que residen en León, Guanajuato; en ella, se analiza la vitalidad de los idiomas amerindios en el contexto urbano, pero tomando en cuenta el proceso de mantenimiento o sustitución que se observa en las comunidades de origen, el cual incide directamente en la práctica y transmisión de los idiomas. Un aspecto que Ilamó la atención cuando se comenzó a trabajar con la gente de Ichán y de Tacuro fue la vitalidad de la lengua purépecha a nivel intergeneracional: se escuchaba a los niños platicando con sus padres y abuelos en este idioma, a pesar de que se considera que en la ciudad había más propensión al desplazamiento lingüístico (Fishman, 1979: 153).

La pregunta de investigación de la que se partió fue: ¿por qué una lengua minorizada, a nivel general, se mantiene y se transmite a través de las generaciones si sus hablantes han sido sujetos de discriminación y son conscientes de los límites que se les han impuesto? La hipótesis que se consideró fue la lealtad étnica o la mayorización étnica, que permite que, aun en situaciones adversas, se mantengan las creencias, valores y prácticas de la cultura propia y que esta se viva en la cotidianidad.

Para hacer acopio de los datos lingüísticos se realizó trabajo de campo en León, Guanajuato, y en Ichán y en Tacuro, Michoacán; en la ciudad se investigó entre agosto de 2015 y abril de 2016, y en los dos pueblos en octubre de 2015 y en mayo de 2016. En primer lugar, se Ilevaron a cabo entrevistas que permitieron abordar aspectos en torno a la vitalidad de la lengua y, sobre todo, sondear las actitudes lingüísticas y conocer los factores que los propios hablantes consideran a favor del purépecha; en segundo, se realizó observación in situ para conocer cuál era el idioma de comunicación a nivel familiar y en los diferentes ámbitos sociales (esto último solo en las comunidades de origen) que son considerados bastión del español: gobierno, educación, salud y religión. 
Una de las observaciones que deben hacerse acerca de los datos lingüísticos concierne a la confiabilidad de estos. Dado que en muchas ocasiones las respuestas de los colaboradores $^{12}$ parecen ser las que consideran que el investigador desea escuchar (Labov, 1972), con el fin de verificar la vitalidad de la lengua se empleó el método de la observación (participativa), esto es, presenciar los contextos cotidianos de la lengua: las transacciones en el mercado, los juegos de los niños, las pláticas en las familias, los diálogos en la calle, los anuncios por los altavoces, etcétera, además de las interacciones comunicativas en la jefatura, el centro de salud, la escuela y los templos.

Las entrevistas realizadas se dividieron en tres bloques: el primero en relación con la adquisición de las lenguas, el segundo tocante a las experiencias de migración y la interacción con la "sociedad nacional", y el tercero respecto de las actitudes lingüísticas. Todas las preguntas fueron abiertas, atendiendo a la naturaleza cualitativa de la investigación. El número de entrevistas estuvo determinado por la información proporcionada por los colaboradores, pues en algunos casos, debido a su insuficiente competencia en español, la respuesta demostraba que no habían comprendido la pregunta ${ }^{13}$. El objetivo fue reunir, al menos, quince respuestas que señalaran los potenciales factores que están a favor del empleo del purépecha, a partir de las cuales se pudiera realizar el estudio.

El análisis de los datos se llevó a cabo a partir de la búsqueda de patrones respecto de los factores que se mencionaron en las entrevistas, tales como la vitalidad de esta lengua indígena y su transmisión intergeneracional. La ideología de los colaboradores, acerca de su idioma, se coligió a partir de considerar las cargas valorativas que se señalaron acerca de esta y se verbalizaron, sobre todo, en calificativos positivos y en posicionamientos casi militantes frente a una posible pérdida y las consecuencias que tendría en su identidad y en su distinción como indígenas en un medio mestizo homogeneizado.

La pregunta de investigación que se planteó acerca de por qué se sigue transmitiendo la lengua purépecha cuando fuera del pueblo, en la ciudad, carece de funciones comunicativas se obtuvo a partir del análisis semántico de las valoraciones de la lengua y la respuesta al motivo por el cual las personas la enseñan a sus hijos. Con el fin de evitar una respuesta sesgada y para obtener una visión panorámica que permitiera observar la realidad lingüística, se conjuntó lo señalado por los dirigentes, profesores y ministros de culto, así como por la gente del pueblo.

12 Prefiero emplear el término "colaborador" en lugar de "informante" de acuerdo con las nuevas tendencias en ciencias sociales y culturales que buscan la "horizontalidad" y el "diálogo" entre el investigador y los sujetos de su estudio (Corona y Kaltmeier, 2012).

13 Hubo también quienes mostraron actitudes desfavorables al idioma indígena, respuestas que no fueron tenidas en cuenta para los propósitos de este estudio. 


\section{El marco sociopolítico, cultural y lingüístico}

Tal y como se mencionó previamente, Ichán y Tacuro se ubican en la cañada de los once pueblos en el estado de Michoacán, junto con Carapan, Huáncito, Zopoco, Santo Tomás, Acachuén, Tanaquillo, Urén, Chilchota y Etúcuaro; los dos citados en primer lugar parecen ser un único pueblo, debido a que se encuentran juntos y parece que existen señalamientos que indican los límites. Cuentan con jefatura, centro de salud, escuelas desde el nivel básico hasta medio superior y templos católicos y evangélicos. La carretera nacional los comunica con el resto del país y pueden desplazarse hacia Zamora, una ciudad cercana de cierta importancia, y Morelia, la capital del estado.

Según se ha señalado, la restricción del uso de una lengua en los ámbitos considerados de prestigio y la consecuente reducción al plano doméstico y a lo no valorado socialmente disminuye sus funciones lingüísticas y puede llevar a la extinción (Junyent, 1995: 37-38). Por todo ello, se decidió observar el uso o desuso del purépecha en aquellos espacios donde el purépecha pudiera estar restringido. El primer espacio fue la administración. En ambos pueblos solo se cuenta con jefatura, pues la cabecera es Chilchota y allí se rigen los destinos del municipio. Las personas responsables de ministrar son originarias de los pueblos y son bilingües, aunque en diferentes grados; algunos cuentan con estudios superiores. Quienes Ilegan a presentar una solicitud o realizar un trámite y hablan purépecha, que son la mayoría, son atendidos en este idioma, por lo cual no existe dificultad en cuanto a la inteligibilidad ni se recurre a un intérprete. La atención que se brinda no muestra signos de asimetría a favor de un idioma y, si hubiera alguna, se inclinaría a favor del purépecha, puesto que es la lengua de uso cotidiano.

En lo que atañe a los servicios de salud, mientras que en Ichán trabajan un médico y una enfermera originarios de la región y hablantes de purépecha — motivo por el cual no se presentan problemas de incomprensión lingüística-, en Tacuro el personal de salud (médico y enfermera) no hablan el idioma, aunque son de la zona purépecha (la zona castellanizada). Quienes deben buscar la manera de hacerse entender y superar la frontera lingüística son los pacientes: generalmente, acuden con un familiar que es bilingüe o recurren a la ayuda de otras personas que se encuentran en la consulta médica. Lo anterior, no obstante, no impele al aprendizaje del español, pues la situación la resuelven de manera fácil y no la consideran en el contexto de una oposición jerárquica.

Por su parte, los servicios religiosos en la Iglesia católica se celebran en español, dado que los sacerdotes que ofician no saben hablar purépecha. Cuando alguna persona, debido a una situación o deseo particular, requiere que se "diga la misa" en el idioma amerindio, solicita la presencia de un cura que sí lo conozca, aunque deba cubrir los gastos que se generan por la visita del sacerdote. En el caso de las iglesias evangélicas, los servicios religiosos se llevan 
a cabo en la lengua indígena - existe una excepción en una iglesia cuyos cultos se realizan en español, debido a que los pastores son de fuera y allí se congregan personas de pueblos que han sido castellanizados-. Según lo que se pudo observar al asistir al Centro Cristiano de Ichán, el purépecha es la lengua de comunicación de y con lo divino; solo se escuchan en español las fórmulas propias de la liturgia evangélica o las citas de la Biblia que se han aprendido de forma memorística.

Según menciona Sáenz (1992 [1936]), Ichán cuenta con escuela desde 1887 y Tacuro desde 1932 ${ }^{14}$. Uno de los objetivos educativos actuales es impartir los cursos en purépecha y enfatizar la importancia de esta cultura, es decir, fomentar la convivencia con el español. Se cuenta que hasta hace unos treinta años la política educativa tendía a la castellanización y se valía de recursos violentos para lograr su fin; sin embargo, la población se organizó y exigió que los profesores fueran de los propios pueblos y hablaran su idioma. De hecho, en la actualidad, en las escuelas primarias de estos dos pueblos el purépecha posee mayor presencia: según se pudo constatar, la mayoría de los niños lo hablan entre sí en todos los espacios y las reuniones de los profesores con los padres de familia (madres, en su mayoría) son en este idioma.

En Ichán y en Tacuro no todos los niños son bilingües cuando llegan a la escuela primaria: los hay monolingües en purépecha y en español. ¿Cómo se resuelve el monolingüismo en uno u otro idioma con ellos? A unos se les enseña purépecha y a otros español con un consiguiente énfasis en el proceso de bilingualización de los niños, dada su continua interacción. El aprendizaje de idioma amerindio por parte de los fuereños es bien valorado en estas comunidades ${ }^{15}$.

Por lo que respecta a los migrantes que radican en León, Guanajuato, la situación les es considerablemente adversa, debido a que los ámbitos de uso del purépecha se reducen allí al hogar y a la convivencia entre paisanos en encuentros de amistad o en actividades de carácter cultural organizadas por instituciones públicas. Algunos niños asisten a una escuela primaria intercultural, de carácter particular, sostenida por jesuitas; en la plantilla docente se encuentran profesores indígenas, hablantes de diferentes idiomas maternos (entre ellos un purépecha), que podrían impartir los cursos en sus respectivas lenguas. Sin embargo, la distinta procedencia etnolingüística tanto del profesorado como del alumnado explica la inviabilidad de tal acción.

14 En su obra, Sáenz (1992 [1936]) realiza un recuento de las condiciones de las escuelas de los pueblos de la Cañada michoacana en tiempo de su proyecto, entre las cuales se encuentran las de Ichán y Tacuro. La presencia del principal agente aculturizante desde entonces en estas dos comunidades indígenas lleva a preguntarse por qué no lograron su cometido.

15 Así, por ejemplo, una mexicana-estadounidense, a quien Ilaman "la gringa”, habla purépecha y es considerada un modelo en el pueblo frente a quienes prefieren hablar solo castellano. 


\section{Análisis de los factores a favor del mantenimiento}

Según se ha señalado y documentado en la bibliografía especializada, entre los factores que han propiciado el desplazamiento de los idiomas amerindios cabe citar la integración en la economía nacional, las decisiones políticas y de agentes gubernamentales, el modelo educativo, los medios de comunicación, así como la construcción de carreteras y otras infraestructuras que permitieron una mejor comunicación entre pueblos (Zimmermann, 2010: 904). Por el contrario, el relativo aislamiento fue determinante en el mantenimiento de lenguas minoritarias y minorizadas (Sáenz, 1992 [1936]; Trujillo y Terborg, 2009: 136-137).

Sin embargo, tal y como señaló Fishman (1979: 149-150), estas variables no siempre conducen a la pérdida de la lengua y pueden propiciar tanto el mantenimiento como el desplazamiento según los contextos donde se produzcan. Así, en los casos de Ichán y de Tacuro los habitantes siguen siendo indígenas (y mexicanos), hablantes de purépecha, aunque se trate de zonas bastante bien comunicadas, que presentan un alto porcentaje de migración —dada la actividad comercial que realizan-, y estén conectadas por internet con el resto del mundo.

Otro caso documentado de mantenimiento del purépecha en situaciones semejantes es el de San José de la Laguna. Según se señaló anteriormente, la vitalidad del idioma en este pueblo se debe a que el dominio lingüístico del hogar no se ha colonizado; por tanto, la lengua de comunicación a nivel familiar es la indígena y, de esta manera, no se interrumpe su transmisión intergeneracional (Rico, 2010); sin embargo, en los pueblos vecinos, distantes a unos pocos kilómetros, ya no se habla purépecha.

De acuerdo con lo que se observó y lo que se mencionó en las entrevistas, el mantenimiento del purépecha se debe, cuando menos, a tres factores: primero, una mayor competencia en el idioma indígena por parte de la generación que está criando hijos o que está en contacto con los niños; segundo, la lealtad étnica o mayorización lingüística, que, a su vez, lleva al tercero, que es el uso de la lengua en ámbitos de prestigio. En el primer caso, destaca el hecho de que existen casos de personas, incluso aquellas que se pueden considerar jóvenes (entre 30 y 40 años, de las cuales algunas ya son abuelos), que únicamente tienen un conocimiento básico de español (algunas entrevistas se dificultaron por este motivo); por tanto, el purépecha es de uso cotidiano en todos los ámbitos ${ }^{16 .}$.

16 Los ejemplos que se presentan son extractos de las entrevistas. Las transcripciones son literales y se sigue la convención de colocar entre paréntesis, por un lado, los puntos suspensivos para indicar que un fragmento intermedio no se incluye y, por otro, las aclaraciones para hacer inteligible la cita. Los textos son representativos y muestran las opiniones y actitudes, semejantes a otras, acerca de la lengua purépecha. 
El primero [idioma] que me aprendió, pues, cuando me acordaba ya hablaba así como mi mamá y papá, así como orita... purépecha. Ya yendo oír a la escuela, ya uno va aprendiendo ya a hablar o escuchar, pues, el español [...]. [Mis papás] hablaban así nomás, como nosotros, así [en purépecha] [...]. Allí [en la escuela] los maestros dicen cosas así, por español y ya uno, poco a poquito, o como yo, no... muchas cosas que todavía no escucho [entiendo] en español; oigo, pues, pero hablar [...]. [Yo enseñé y hablo con mis hijos en purépecha] porque lo mismo que no hablábamos nosotros [en español] y ya empiezan a hablar como nosotros [en purépecha]. (Mujer, 36 años, ama de casa - comerciante. Entrevistada el 20 de mayo de 2016).

El segundo factor se observó en las personas bilingües que podrían enseñar únicamente español a sus hijos pero que, por cuestiones de aprecio de su lengua materna, prefieren socializar a sus hijos en purépecha y tenerlo como idioma en la cotidianidad. El empleo del español tiene un carácter instrumental, pues se ven en la necesidad de comunicarse en este idioma con el resto de la población no purepechahablante:

Aquí en la casa y con todos los conocidos [hablo] purépecha; y ya, por ejemplo, con la enfermera, el doctor y con los que hice el servicio [social] que nos hablamos en la escuela, en la prepa, español. [...] Toda la Cañada [de los once pueblos] casi habla el purépecha y yo por eso se lo enseñaría [a mi hijo] porque, por ejemplo, si entra aquí a la escuela, a la prepa de Ichán, casi todos hablan el purépecha; entonces, se puede comunicar con los alumnos; y ya, con los maestros, en español. (Mujer, 21 años, ama de casa - estudiante. Entrevistada el 19 de mayo de 2016).

La lealtad étnica, la persistencia en la cultura propia, desempeña un papel fundamental, puesto que implica la defensa, práctica y transmisión de la cultura sobre todo en situaciones adversas para los portadores de la misma. Así lo expresó una señora originaria de Tacuro que vive en León:

Aunque a mí me trataran mal yo no voy a dejar de hablar [purépecha] y que mis hijos también y mis nietos hablen el idioma. No. ¿Por qué ya no voy a querer que [mis descendientes lo] hablen? No, pues [con] más [razón lo hablaré]. A mí, como que me echaran fuego... [cuando se menciona que se debe dejar de hablar]. No, yo no [lo haré]. Dijera, iay, me están maltratando! No, al contrario, con más ganas enseñaría a mis hijos... o enseñé. (Mujer, 53 años, ama de casa - comerciante. Entrevistada el 14 de marzo de 2016).

El tercer factor es la comunicación en purépecha en todos los espacios de la vida cotidiana, incluyendo aquellos que son considerados de prestigio: escuchar la "palabra de Dios" en su propio idioma confiere a este una valoración positiva; de igual manera, que la educación se imparta en la lengua indígena le brinda prestigio:

Aquí tratamos de enseñar en lengua purépecha, explicar en lengua purépecha; aunque sean contenidos oficiales, pero tratamos de explicar en lengua purépecha; lo explicamos de hecho en lengua 
purépecha y después en español; la mayoría de las asignaturas las explicamos en lengua purépecha. Forzosamente [debemos hablar purépecha los cinco días de clase en purépecha], es la lengua materna de aquí y ellos [los niños] vienen y me preguntan en purépecha, no me preguntan en español. [Los alumnos] dominan más el purépecha. (Hombre, 30 años, profesor. Entrevistado el 21 de mayo de 2016).

En el caso del centro de salud donde los encargados solo hablan español no se producen presiones para que se produzca el aprendizaje de este, pues lo que se prioriza es la comunicación e inteligibilidad en cualquiera de las dos de la manera como sea posible, como se mencionó; además, se presentan casos de jóvenes que, aunque sean bilingües, cuando acuden a la consulta únicamente hablan purépecha:

Sí sería ideal tener aquí a alguien que manejara la lengua [purépecha]. [...] La mayoría [de los jóvenes] tiene el dominio de los dos idiomas y es más fácil que te puedan comprender. [...] Hay otros [jóvenes] que son muy orgullosos y aunque hablan el español no te lo manifiestan. Eso es algo que también noté acá: el orgullo del imperio purépecha es muy... No es como apatía, no; no es algo que tú digas es un problema social, sino que ellos son así, son reservados, se apropian del idioma. (Hombre, 28 años, médico. Entrevistado el 18 de mayo de 2016).

Podría parecer que las personas que por su mayor competencia en purépecha enseñan esta lengua a sus hijos lo hacen únicamente por esta razón; sin embargo, en esta decisión se manifiesta una intención de mantenimiento que contrasta con la situación de desplazamiento; en este último caso, en cuanto las personas adquieren una mínima competencia oral en español que les permita comunicarse, empiezan a enseñar este idioma a sus hijos: el tránsito de una lengua a otra, derivado del aprendizaje de la segunda, es evidente en el idioma que aprenden sus hijos, según el orden de su nacimiento: los mayores aprenden el idioma amerindio y los menores español, con gradación de bilingüismo, sobre todo, entre los intermedios (Canuto, 2015).

Este hecho contrasta de igual manera con lo que se ha observado en otros pueblos en los que se ha producido desplazamiento lingüístico: cuando la mayoría de la población hablaba el idioma amerindio, los cultos en las iglesias evangélicas se realizaban en este idioma, pero, conforme avanzó la castellanización, la lengua indígena fue desplazada de los templos. A nivel general, entre los indígenas pocas personas mencionan que su idioma no es útil y que no vale la pena enseñarlo a sus hijos; casi todos dan una valoración positiva a su lengua por ser herencia de sus antepasados, muestran pesar porque se está perdiendo y cuando se les pregunta por qué los niños no saben su idioma responden que se debe a que no quieren aprenderlo. En Ichán y en Tacuro también se mencionaron los mismos argumentos a favor de la transmisión del purépecha:

Es una lengua original de nosotros, que no debemos... cambiarla por el español... porque somos purépechas. Por eso [hablamos purépecha]. [...] A veces digo que no está bien que los mismos de 
aquí le enseñen a sus hijos [únicamente] español [...] y es como se va olvidando el purépecha. [...] Es como una herencia de nuestros antepasados que empezaron a hablarlo; entonces, sería feo que nada más porque todos hablan español nosotros también nos vamos a cambiar, a hablar puro español. (Mujer, 22 años, ama de casa - comerciante. Entrevistada el 19 de mayo de 2016).

\section{Conclusión}

¿Qué variables explican en unos el mantenimiento y en otros el desplazamiento lingüístico? Para responder a esta cuestión se proponen dos niveles de acción frente a un proceso de minorización desde afuera: el primero corresponde a la conciencia étnica, que es la capacidad de aprehender la situación lingüística y las implicaciones para el hablante y su cultura; el segundo, la lealtad étnica, indica una posición activa a favor, a pesar de lo adverso del contexto. Así, la voluntad de mantener la lengua se pondera a partir de la conciencia étnica en oposición a las presiones que se ejercen para la castellanización: si se cede ante los embates por convencimiento de un supuesto beneficio, se producirá el desplazamiento debido a la minorización efectiva; si, por el contrario, se decide que a pesar de los factores en contra se hablará el idioma indígena y se enseñará a los niños, es porque ha cobrado importancia la identidad y lealtad étnicas: se ha producido la mayorización del propio idioma y se le ha colocado en el mismo nivel de prestigio que otros mayorizados como el español.

Cuando los hablantes de lenguas indígenas se manifiestan a favor de estas en el discurso pero en la praxis se observa lo contrario, esta actitud no debe considerarse ambivalente o contradictoria. Se trata en muchas ocasiones de una decisión razonada surgida de ponderar los deseos de mantenimiento y las presiones ejercidas sobre ellos. Así, la disyuntiva parece encontrarse entre decidir por el "amestizamiento" (hacerse como mestizo, pero seguir siendo indígena) y la "amestización” (hacerse mestizo): la primera implica apropiarse de elementos culturales de manera funcional para vivir en un mundo que se ha vuelto mestizo mayoritariamente (lo cual no remite necesariamente a una mezcla biológica, sino a la adquisición de la cultura que se considera hegemónica), mientras que la segunda significa abandonar la cultura indígena, desindianizarse, y hacer propia la del grupo dominante. Es una cuestión de conciencia y voluntad.

¿Por qué conservar o no una lengua indígena? La respuesta está determinada por la decisión de sus hablantes como consecuencia de su autoconstrucción social y étnica, y en ella se encuentra la calidad que dan a su idioma: minorizado o mayorizado. En un contexto como el mexicano, donde el desplazamiento lingüístico es lo más común, se encuentran los casos de Ichán y Tacuro, que han decidido mantener el idioma purépecha y lo han logrado porque, colectivamente, poseen conciencia étnica y han dado a su idioma la calidad de mayorizado. 


\section{Bibliografía citada}

Aguirre Beltran, Gonzalo, 1992 [1976]: Obra antropológica XI. Obra polémica, México: Universidad Veracruzana / Instituto Nacional Indigenista / Gobierno de Veracruz / Fondo de Cultura Económica.

Brice Heath, Shirley, 1992 [1970]: La política del lenguaje en México. De la Colonia a la nación, México: Consejo Nacional para la Cultura y las Artes / Instituto Nacional Indigenista.

CAlvet, Louis-Jean, 2005 [1974]: Lingüística y colonialismo. Breve tratado de glotofagia, México: Fondo de Cultura Económica.

Canuto Castillo, Felipe, 2013: "Las lenguas indígenas en el México de hoy. Realidad y política lingüísticas”, Lenguas Modernas 42, 31-45.

Canuto Castillo, Felipe, 2015: "Otomíes en la ciudad de México. La pérdida de un idioma en tres generaciones”, Lengua y Migración 7-1, 53-81.

Comisión Nacional para el Desarrollo de los Pueblos Indigenas (CDI), 2006: "Percepción de la imagen del indígena en México" [http://www.cdi.gob.mx/dmdocuments/percepcion_imagen_indigena_mexico.pdf, fecha de consulta: 01 de mayo de 2016].

Corona, Sarah, y Olaf Kaltmeler (coords.), 2012: En diálogo. Metodologías horizontales en ciencias sociales y culturales, Barcelona: Gedisa.

FIsHman, Joshua, 1979: Sociología del lenguaje, Madrid: Cátedra.

HekkIng, Ewald, 1995: El otomí de Santiago Mexquititlán: desplazamiento lingüístico, préstamos y cambios gramaticales, Amsterdam: IFOTT.

Instituto Nacional IndigenISTA (INI), 1962: Los centros coordinadores, México: INI.

Junyent, Carme, 1995: Vida i mort de les /lengües, Barcelona: Empúries.

Labov, William, 1972: Language In The Inner City: Studies In The Black English Vernacular, Philadelphia: University of Pennsylvania Press.

Loyo, Engracia, 1996: “La empresa redentora. La casa del estudiante indígena”, Historia mexicana, vol. 46, núm. 1, 99-131.

NavarRete, Federico, 2004: Las relaciones interétnicas en México, México: Universidad Nacional Autónoma de México.

Ninyoles, Rafael, 1975: Estructura social y política lingüística, Valencia: Fernando Torres, Editor. 
Rıco, Gabriel, 2010: Mantenimiento y resistencia de la lengua p’urhèpecha en Santa Fe de la Laguna, Michoacán. Tesis de maestría, Universidad Nacional Autónoma de México.

SAenz, Moisés, 1992 [1936]: Carapan [http://www.crefal.edu.mx/crefal25/images/publicaciones/libros/carapan.pdf, fecha de consulta: 01 de mayo de 2016].

TeRBorg, Roland, y Laura García LANDA (coords.), 2011: Muerte y vitalidad de las lenguas indígenas y de las presiones sobre sus hablantes, México: Universidad Nacional Autónoma de México.

TRUjILlo, Isela, y Roland Terborg, 2009: "Un análisis de las presiones que causan el desplazamiento o mantenimiento de una lengua indígena de México. El caso de la lengua mixe de Oaxaca”, Cuadernos Interculturales, vol. 7, núm. 12, 127-140.

URAnGA, Belén, y Maider Magaña (eds.), 2008: El futuro de las lenguas. Diversidad frente a la uniformidad, Madrid: Catarata.

Villavicencio, Frida, 2013: Lenguas indígenas en el México decimonónico. Ecos, pregones y contrapuntos, México: Centro de Investigaciones y Estudios Superiores en Antropología Social.

Villoro, Luis, 2014 [1950]: Los grandes momentos del indigenismo en México, México: Fondo de Cultura Económica.

Wright, David, 2007: “La política lingüística en la Nueva España”, Acta Universitaria, vol. 17, núm. 3, 5-19.

ZimmeRmann, Klaus, 1992: Sprachkontakt, ethnische Identität und Identitätsbeschädigung: Aspekte der Assimilation der Otomí-Indianer an die hispanophone mexikanische Kultur, Frankfurt: vervuert.

Zimmermann, Klaus, 2010: "Diglosia y otros usos diferenciados de lenguas y variedades en el México del siglo XX. Entre el desplazamiento y la revitalización de las lenguas indomexicanas" en Rebeca Barriga y Pedro Martín Butrageño (eds.): Historia sociolingüística de México, vol. II, México: El Colegio de México, 881-955. 